\title{
An iterative AC-SCOPF approach managing the contingency and corrective control failure uncertainties with a probabilistic guarantee
}

\author{
Efthymios Karangelos, Member, IEEE, and Louis Wehenkel
}

\begin{abstract}
This paper studies an extended formulation of the Security Constrained Optimal Power Flow (SCOPF) problem, which explicitly takes into account the probabilities of contingency events and of potential failures in the operation of post-contingency corrective controls. To manage such threats, we express the requirement that the probability of maintaining all system operational limits, under any circumstance, should remain acceptably high by means of a chance-constraint. Further, representing power flow as per the full $\mathrm{AC}$ model, we propose a heuristic solution approach leveraging state-of-the-art methodologies and tools originally developed to tackle the standard, deterministic-constrained SCOPF statement. We exemplify the properties of our proposal by presenting its application on the three area version of the IEEE-RTS96 benchmark, stressing the interpretability of both the chance-constrained reliability management strategy and of the heuristic algorithm proposed to determine it. This work serves to showcase that the first step on the transition towards probabilistic reliability management can be achieved by suitably adapting presently available operational practices and tools.
\end{abstract}

Index Terms-Reliability management, AC-SCOPF, chanceconstraint, contingency probability, corrective control failure, iterative decomposition.

\section{INTRODUCTION}

W ITH a view in enabling the evolution from the $\mathrm{N}-1$ deterministic practice to a risk-based probabilistic approach, the (un)availability of data, the (challenging) interpretability of methods and of the results thereof and the (increased) computational complexity constitute the modern priorities for research and development in power system reliability management [1]. Anticipating the gradual progress on data availability, considerable effort is targeting the probabilistic variant of the Security Constrained Optimal Power Flow (SCOPF) problem [2]. In particular, the definition of appropriate metrics to express the risk implied by credible contingencies, the integration of such metrics within the 'classical' SCOPF decision framework and the efficient algorithmic solution of the mathematical problem resulting from choices made regarding the aforementioned are open research topics.

Integrating the expected cost of corrective control in the ACSCOPF objective function, Xu et al. [3] developed a solution strategy composed of a global search for the upper bounds on "critical" decision variables and a local search for an optimal solution given such boundaries. Capitanescu proposed an ACSCOPF constraining the expected post-contingency voluntary

Efthymios Karangelos and Louis Wehenkel are with the Department of Electrical Engineering \& Computer Science - Institut Montefiore, University of Liège, Liège, Belgium, email: \{e.karangelos,l.wehenkel\}@uliege.be. load-shedding and established the solvability of medium-scale problem instances while relying on a standard Non-Linear Programming (NLP) solver [4]. Shchetinin and Hug [5] stated an alternative AC-SCOPF problem while constraining the total risk implied by an ensemble of single-order and doubleorder outages expressed in function of the post-contingency component loading, and developed a sensitivity-based iterative decomposition approach. Risk was also expressed in terms of expected component loading in [6], wherein the effectiveness of coupling Lagrangian relaxation and Benders decomposition to solve the DC-approximation of the steady-state SCOPF problem was established.

All the aforementioned works model post-contingency corrective controls as perfectly fail-safe. Several European Transmission System Operators (TSOs) consider the lack of modeling the possible failure of corrective controls as a noteworthy deficit in existing tools [7]. In practice, the realization of hidden failures in protection, controls and communications only after a single initiating disturbance is a well documented factor in many observed blackouts [8] which also suggests that the effectiveness of post-contingency corrective controls should be considered as uncertain. Reference [9] provides a framework for modeling corrective control failure in the context of reliability assessment studies as well as a comprehensive discussion of necessary improvements in terms of data. Further, in [10] we presented a comparative analysis on the relevance of corrective control failure probability and risk under the N-1 operational regime. Finally, in our earlier works we progressively developed a probabilistic reliability management approach consisting of (i) an adaptive contingency set, and (ii) a chance-constrained SCOPF explicitly acknowledging the potential failure of post-contingency corrective controls [11], [12]. We demonstrated the principles of this proposal while relying on the DC-power flow approximation, which makes medium-scale instances of the resulting problem directly solvable by classical solvers.

In the present paper we revisit the SCOPF component of the framework proposed in [12] and refine its statement while integrating the non-linear AC power flow model. Acknowledging that such modeling enhancement comes at a notable increase of computational complexity, we focus on establishing the solvability of the resulting decision-making problem. We leverage the state-of-the-art iterative decomposition ideas developed for the deterministic-constrained SCOPF problem in [13]-[15] and extend its applicability to the probabilistic, chance-constrained problem. To do so, we propose (i) an 
expanded scope for the contingency assessment module of the iterative decomposition SCOPF solution approach to take into account both corrective control and its failure probability, and, (ii) probability and risk aware contingency filtering variants, targeting the feasibility of the probabilistic, chance-constrained statement. We establish the functionality of such methodological adaptations by means of demonstrative case studies and investigate the implications of the proposed alternative contingency filtering variants, showcasing the interpretability of our overarching proposal. This work serves as a first step to expose the broad potential for further developing existing algorithmic solutions approaches \& operational practice to enable the transition to probabilistic reliability management.

The rest of this paper is structured as follows: Section II provides the mathematical formulation of the problem tackled, while Section III describes our algorithmic solution approach, Section IV] our case studies on the IEEE RTS-96 3-area system, and Section $\mathrm{V}$ draws our conclusions.

\section{Problem Formulation}

To establish a frame of reference and introduce notational conventions, let us begin by reproducing from [16] the compact statement of the deterministic-constrained SCOPF problem as per the state-of-the art:

$$
\begin{array}{rl}
\min _{\mathbf{u} \in \mathbf{U}} & C P\left(x_{0}, u_{0}\right) \\
& h_{0}\left(x_{0}, u_{0}\right) \leq 0 ; \\
& h_{c}^{s}\left(x_{c}, u_{0}\right) \leq 0 \quad \forall c \in \mathcal{C} ; \\
& h_{c}\left(x_{c}^{w}, u_{c}\right) \leq 0 \quad \forall c \in \mathcal{C} ; \\
& \mathbf{u} \in \mathbf{U} \equiv\left\{u_{0} \in \mathcal{U}_{0}\left(x_{0}\right) ; u_{c} \in \mathcal{U}_{c}\left(x_{0}, u_{0}, c\right) \forall c \in \mathcal{C}\right\} .
\end{array}
$$

Throughout 2,4 we denote by $h .(x, u$.) the group of equality and inequality constraints expressing a viable steadystate of the system in function of state variables $x$. (i.e., voltage magnitude and phase angle) and decision variables $u$. (e.g., generator active power dispatch and voltage set-points, etc.). More specifically $h .(x ., u) \leq$.0 refers to the AC power flow equations, as well as upper and lower bounds on voltage magnitude per node, and on voltage angle difference and apparent power flow per branch. Constraint group (2) and subscript 0 concern the operation of the intact system, prior to the occurrence of any credible contingency $c \in \mathcal{C}$. To protect against any such eventuality, group (3) expresses the temporary viability of the system immediately following the contingency (i.e., at the so-called 'intermediate' post-contingency stage) but still prior to the application of corrective controls. Notice that in (3) decision variables maintain the values set for precontingency operation while superscript $(s)$ expresses the tolerable temporary relaxation of operational constraints. Finally, group (4) reinstates the permanent system operational limits by means of post-contingency corrective controls (denoted by decision variables $u_{c}$ ). We use superscript $(w)$ in (4) to represent that (1-5) assumes that corrective controls are fully fail-safe and always work as expected. In other words, it disregards uncertainty in the corrective control effectiveness. Bounds on controllable resources, including the range of admissible deviations between corrective and preventive controls are represented by (5).

\section{A. Probabilistic, chance-constrained SCOPF statement}

As already mentioned, in this work we revisit the SCOPF component of the probabilistic framework developed in our earlier work [12]. Referring the reader also to [17] for a detailed exposition of the features of such framework, let us introduce the compact statement of the probabilistic, chanceconstrained SCOPF as in (6) 9 .

$$
\begin{array}{rl}
\min _{\mathbf{u} \in \mathbf{U}} & C P\left(x_{0}, u_{0}\right)+\sum_{c \in \mathcal{C}} \pi_{c} \cdot C C\left(x_{0}, u_{0}, c, u_{c}\right) ; \\
& h_{0}\left(x_{0}, u_{0}\right) \leq 0 \\
& \mathbb{P}\left\{\begin{array}{c}
h_{c}^{s}\left(x_{c}, u_{0}\right) \leq 0 \\
h_{c}\left(x_{c}^{b}, u_{c}\right) \leq 0
\end{array} \mid(c, b) \in \mathcal{C} \times \mathcal{B}\right\} \geq 1-\epsilon ; \\
& \mathbf{u} \in \mathbf{U} \equiv\left\{u_{0} \in \mathcal{U}_{0}\left(x_{0}\right) ; u_{c} \in \mathcal{U}_{c}\left(x_{0}, u_{0}, c\right) \forall c \in \mathcal{C}\right\} .
\end{array}
$$

Problem (6) is a generalization of 1,51 by taking into account (i) the contingency probabilities $\left(\pi_{c}\right)$, and, (ii), the costs of post-contingency corrective controls $\left(C C\left(x_{0}, u_{0}, c, u_{c}\right)\right)$, and notably, (iii) the elements $b$ of set $\mathcal{B}$ representing the possible outcomes of post-contingency corrective controls (e.g., fully working, partially working, fully failing, etc.). Chance constraint (8) is the security constraint replacing groups (3-4) to enforce that, subject to the combined uncertainty on the contingency occurrence and on the postcontingency corrective control outcome (denoted as $(c, b) \in$ $\mathcal{C} \times \mathcal{B})$ ), the system should remain functional with probability at least equal or greater than an acceptable lower bound $(1-\epsilon)$. In other words, the lower bound set by chance constraint (8) should be interpreted as an explicit degree of confidence on keeping the system within all relevant operational limits.

\section{B. Mathematical models \& assumptions}

In our implementation we used the following models and assumptions, while representing steady state power flow by the AC model in rectangular coordinates.

a) Control variables: We model the generator active power dispatch and voltage set-points as control variables, defined in the preventive stage and re-defined per contingency for the corrective post-contingency stage. At all problem stages we model lower $\&$ upper bounds on active and reactive power generation and voltage magnitude. For the intermediate postcontingency stage, we model a distributed slack bus and adjust active power generation through fixed participation factors. At this stage of the problem, we constrain the voltage magnitude at generator buses to the preventive set-points and neglect the additional degree of freedom offered by PV/PQ switching. Finally, we consider upward and downward active power ramping restrictions between the preventive and any postcontingency corrective generation dispatch.

b) Network operational limits: We impose for all problem stages lower and upper bounds on voltage magnitude per node, on voltage angle difference per branch as well as on apparent power flow limits per branch. The latter are less restrictive in the intermediate post-contingency problem stage.

\footnotetext{
${ }^{1}$ With the appropriate choice of numerical parameters, problem 669 is indeed reducible to 1,5 .
} 
c) Contingency probabilities: We rely on the fundamental model of [18] and express the outage probability of occurrence in function of the respective outage rate. The pseudo-contingency of no-outage $\left(c_{0}\right)$ is accounted for with occurrence probability equal to $\pi_{c_{0}}=1-\sum_{c \in \mathcal{C} \backslash c_{0}} \pi_{c}$.

d) Objective function: Without loss in generality, we adopt as a minimization objective the aggregation of preand post-contingency corrective upward generation re-dispatch marginal costs. The latter is integrated as a probabilityweighted summation over the contingency set, while assuming that it is payable irrespective of the corrective control effectiveness.

e) Corrective control possible outcomes: We assume that upon occurrence of any contingency $c \in \mathcal{C}$, the corresponding pre-selected set of elementary control operations $\left(u_{c}\right)$ may either work effectively or not. To elaborate on the latter eventuality, we first note that in the context of a SCOPF problem post-contingency corrective controls are chosen without any redundancy. In other words, the complete set of many elementary control operations (e.g., re-setting of the voltage set-points and re-dispatching of the active power output of several units, changing of several breakers, transformer taps, etc.) needs to be successfully implemented in a timely manner to safely alleviate constraint violations [19]. It follows that the failure of any single elementary control operation would result in failing to address constraint violations on time, thus making corrective control ineffective. On this basis, we model the possible outcome of corrective control as a discrete, twomember set $b \in \mathcal{B}=\{w ; f\}$ corresponding to the working $(w)$ and failing $(f)$ realizations [11] and denote the respective probability as $\pi_{c}^{b}$.

f) Chance constraint reformulation: Working with discrete sets of contingencies and corrective control outcomes, we equivalently express chance constraint 8 as,

$1-\sum_{c \in \mathcal{C}} \pi_{c} \sum_{b \in \mathcal{B}} \pi_{c}^{b} \cdot \mathcal{I}\left(x_{0}, u_{0}, c, u_{c}, b\right) \geq 1-\epsilon$

where indicator function $\mathcal{I}\left(x_{0}, u_{0}, c, u_{c}, b\right)$ takes the value of one only in the event that the combination of pre-contingency stage, controls, contingency, and corrective control outcome is not viable and leads to operational constraint violations, as denoted $\forall c, b \in \mathcal{C} \cdot \mathcal{B}$, by the $1^{\text {st }}$ row in (11). Alternatively, as per the $2^{\text {nd }}$ row of $(11)$, it takes the zero value.

$\mathcal{I}\left(x_{0}, u_{0}, c, u_{c}, b\right)=\left\{\begin{array}{l}1 \equiv\left\{h_{c}^{s}\left(x_{c}, u_{0}\right) \not \leq 0 \vee h_{c}\left(x_{c}^{b}, u_{c}\right) \leq \leq 0\right\} \\ 0 \equiv\left\{h_{c}^{s}\left(x_{c}, u_{0}\right) \leq 0 \wedge h_{c}\left(x_{c}^{b}, u_{c}\right) \leq 0\right\}\end{array}\right.$.

Constraint 10 enforces that the aggregate probability of all events (i.e., contingencies and corrective control outcomes) under which operational constraints remain valid is ensured to be acceptably high. It sets the minimum size of the probability space of those contingencies that should be covered by the failsafe preventive controls. For a practical example, considering that the conventional operational constraints of the SCOPF problem express a steady-state equilibrium with no loss of load, constraint 10 would mean that the probability of a situation wherein load is shed involuntarily is at most as small as $(\epsilon)$.

In principle, indicator function $\mathcal{I}\left(x_{0}, u_{0}, c, u_{c}, b\right)$ can be included in the problem statement in complementarity with the respective post-contingency constraints. In [12] we made use of binary indicator variables and the big- $M$ reformulation technique to model the disjunctive inequalities in 11 while exploiting the linearity of the DC approximation to state and solve a Mixed-Integer Linear Programming (MILP) problem via branch-and-bound. Pursuing such strategy while integrating the full AC power flow model would yield a large non-convex Mixed-Integer Nonlinear Programming (MINLP) problem. In principle, the generalized Benders decomposition approach is an applicable solution technique for such a problem. However, further for the question of efficiently handling the big- $M$ parameter, we underline that the problem features a coupling constraint between all $2^{\text {nd }}$-stage discrete and continuous variables (10]. As discussed in [5], for a riskbased SCOPF problem of this form including the non-convex AC power flow model, relying on Benders decomposition may be a rather inefficient approach. Further motivated by results in [20], [21] on the merits of the column \& constraint generation decomposition with respect to the solvability of the 'master' problem and number of iterations, we develop in this paper an alternative heuristic approach adapting the main philosophy of column \& constraint generation to solve the chance-constrained problem (6,9).

\section{ALGORITHMIC SOLUTION APPROACH}

We propose an iterative decomposition approach along the ideas developed in [13]-[15]. The approach seeks to achieve a feasible solution to the chance-constrained statement (6-9) by progressively growing the subset of contingencies to be explicitly covered by a (simpler) deterministic-constrained SCOPF problem, enforcing (2 5). Doing so is enabled by the premise that defining a subset of contingencies to be explicitly covered by a deterministic-constrained SCOPF problem effectively sets a lower bound on the probability of keeping the system within its operational limits appearing on the left-hand-side of (8).

\section{A. Overview}

Let us consider partitioning the contingency set $\mathcal{C}$ to define, $\mathcal{C}_{\mathcal{C}} \subseteq \mathcal{C}: \quad$ a subset of correctively secured contingencies, to be explicitly covered by a determinstic-constrained SCOPF by means of corrective and preventive controls;

$\mathcal{C}_{\mathcal{P}} \subseteq\left(\mathcal{C} \backslash \mathcal{C}_{\mathcal{C}}\right)$ : a subset of preventively secured contingencies, to be explicitly covered by a deterministic-constrained SCOPF only via preventive controls;

$\mathcal{C}_{\mathcal{X}} \subseteq \mathcal{C}: \quad$ the complement of the former two $\left(\mathcal{C}_{\mathcal{X}}:=\mathcal{C} \backslash\left(\mathcal{C}_{\mathcal{C}} \cup \mathcal{C}_{\mathcal{P}}\right)\right)$, composed of all contingencies not explicitly covered by the deterministic-constrained SCOPF.

Any such partition effectively sets a lower bound on the probability of maintaining the system functionality (i.e., the left-hand-side of (8)) computable by adding up the probability 


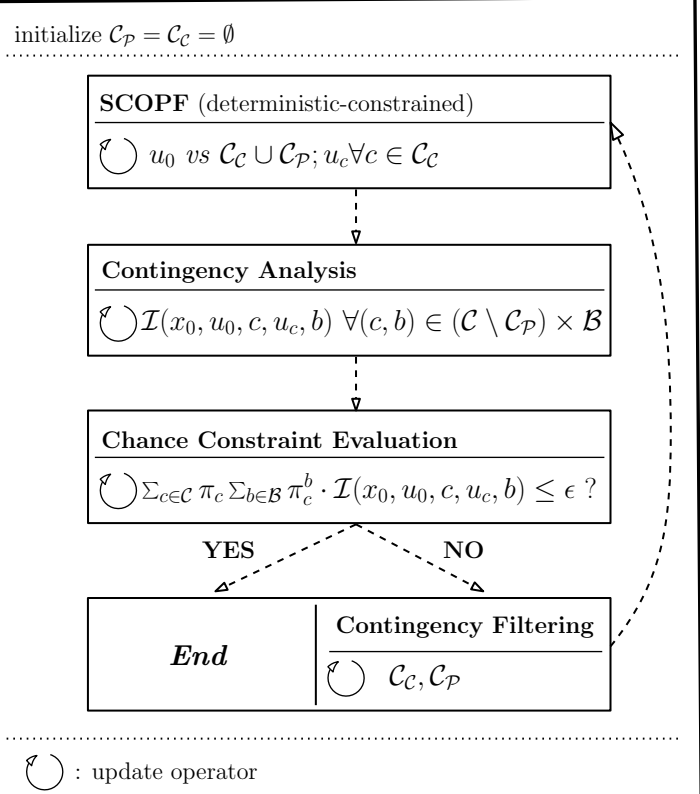

Fig. 1. Iterative solution approach overview

of realizing any contingency $c \in \mathcal{C}_{\mathcal{X}}$, or the failure of corrective controls following any contingency $c \in \mathcal{C}_{\mathcal{C}}$ as,

$1-\left(\sum_{c \in \mathcal{C}_{\mathcal{X}}} \pi_{c}+\sum_{c \in \mathcal{C}_{\mathcal{C}}} \pi_{c} \cdot \pi_{c}^{f}\right)$

where symbol $\pi_{c}^{f}$ denotes the probability of corrective control failure. Indeed, any contingency $c \in \mathcal{C}_{\mathcal{X}}$ may conceivably lead to operational constraint violations and corrective controls could possibly fail for any contingency $c \in \mathcal{C}_{\mathrm{d}}{ }^{2}$. In contrast, preventively secured contingencies $c \in \mathcal{C}_{\mathcal{P}}$ should not in any case result in operational constraint violations, as preventive controls are considered perfectly fail-safe. It follows that growing subsets $\mathcal{C}_{\mathcal{C}}, \mathcal{C}_{\mathcal{P}}$ pushes the lower bound for the lefthand-side of (8) upwards and can be used to progressively guide the deterministic-constrained problem towards a feasible solution of the chance-constrained one (6 9 , by enforcing that the preventive controls are chosen so as to at least safeguard an adequate part of the uncertainty space.

The overview of the resulting solution approach is presented in Fig 1 Initializing subsets $\mathcal{C}_{\mathcal{C}}, \mathcal{C}_{\mathcal{P}}$ as empty, we employ:

- a deterministic-constrained SCOPF solution module to update preventive and corrective controls $v s$ predefined subsets of contingencies to be explicitly covered;

- a contingency analysis module to update the value of indicator function (11) for all contingencies that are not preventively secured;

- a chance constraint evaluation module to update the probability of maintaining the system within all operational limits;

\footnotetext{
${ }^{2}$ In practice of course, several contingencies $c \in \mathcal{C}_{\mathcal{X}}$ may be implicitly covered and also some contingencies $c \in \mathcal{C}_{\mathcal{C}}$ may actually require preventive controls only, making the potential failure of corrective controls ineffectual.
}

- a contingency filtering module to update the subsets of contingencies to be explicitly covered by the deterministic-constrained SCOPF.

\section{B. Deterministic-constrained SCOPF}

For given subsets of contingencies to be explicitly covered, we solve problem $13-18$ to update control actions while neglecting at this preliminary stage the potential failures of corrective controls. Notice that, for preventively secured contingencies we enforce the more strict requirements (15) while for correctively secured contingencies the groups 17) apply.

$$
\begin{aligned}
& \min _{\mathbf{u} \in \mathbf{U}} C P\left(x_{0}, u_{0}\right)+\sum_{c \in \mathcal{C}_{\mathcal{C}}} \pi_{c} \cdot C C\left(x_{0}, u_{0}, c, u_{c}\right) ; \\
& h_{0}\left(x_{0}, u_{0}\right) \leq 0 ; \\
& h_{c}\left(x_{c}, u_{0}\right) \leq 0 \quad \forall c \in \mathcal{C}_{\mathcal{P}} ; \\
& h_{c}^{s}\left(x_{c}, u_{0}\right) \leq 0 \quad \forall c \in \mathcal{C}_{\mathcal{C}} ; \\
& h_{c}\left(x_{c}^{w}, u_{c}\right) \leq 0 \quad \forall c \in \mathcal{C}_{\mathcal{C}} ; \\
& \mathbf{u} \in \mathbf{U} \equiv\left\{u_{0} \in \mathcal{U}_{0}\left(x_{0}\right) ; u_{c} \in \mathcal{U}_{c}\left(x_{0}, u_{0}, c\right) \forall c \in \mathcal{C}_{\mathcal{C}}\right\} .
\end{aligned}
$$

\section{Contingency analysis}

Keeping the preventive and corrective control decisions fixed $\left(\bar{u}_{0}, \bar{u}_{c} \forall c \in \mathcal{C}_{\mathcal{C}}\right)$ as per the latest solution of the deterministic-constrained SCOPF problem we seek to assess the status of post-contingency constraint groups under the working and failing outcomes of corrective control. To perform the respective feasibility checks we use non-negative continuous variables corresponding to fictitious slack generation and demand of active and reactive power at each network node, as per [22]. Let us compactly denote vectors of fictitious nodal slack variables relaxing the nodal active \& reactive power equality constraints as $\delta_{c}^{s}$ for the intermediate post-contingency stage, $\delta_{c}^{w}$ for the corrective-working post-contingency outcome and $\delta_{c}^{f}$ for the corrective-failing instance.

For any contingency not explicitly covered by the deterministic-constrained SCOPF $\left(c \in \mathcal{C}_{\mathcal{X}}\right)$, we solve OPF sub-problem (19) 23 to evaluate whether it is manageable by corrective controls. Notice that the objective in 19 seeks to minimize the introduced relaxation variables. Accordingly, non-zero optimal values for slack variables $\left(\delta_{c}^{s, \star}, \delta_{c}^{w, \star}\right)$ denoting that slack generation and/or demand is necessary to keep the system within operational limits imply that the respective post-contingency state is indeed infeasible

$$
\begin{gathered}
\min _{\delta_{c}^{s}, \delta_{c}^{w}} \mathbf{1}^{T}\left(\delta_{c}^{s}+\delta_{c}^{w}\right) \\
h_{c}^{s}\left(x_{c}, \bar{u}_{0}\right) \leq \delta_{c}^{s} ; \\
h_{c}\left(x_{c}^{w}, u_{c}\right) \leq, \delta_{c}^{w} ; \\
u_{c} \in \mathcal{U}_{c}\left(x_{0}, \bar{u}_{0}, c\right) ; \\
\delta_{c}^{s}, \delta_{c}^{w} \geq 0 .
\end{gathered}
$$

Further, for any contingency not preventively secured by the deterministic-constrained $\operatorname{SCOPF}\left(c \in \mathcal{C} \backslash \mathcal{C}_{\mathcal{P}}\right)$, we follow a

\footnotetext{
${ }^{3}$ The assessment is trivial for any other contingency already explicitly covered by the SCOPF, with $\delta_{c}^{s, \star}=\delta_{c}^{w, \star}=0, \quad \forall c \in \mathcal{C}_{\mathcal{C}} \cup \mathcal{C}_{\mathcal{P}}$.
} 
similar practice and also solve OPF sub-problem 2426) to evaluate the impact of a potential corrective control failure 4

$$
\begin{aligned}
\min _{\delta_{c}^{f}} \mathbf{1}^{T} \delta_{c}^{f} \\
\\
h_{c}\left(x_{c}, \bar{u}_{0}, \delta_{c}^{f}\right) \leq 0 \\
\delta_{c}^{f} \geq 0 .
\end{aligned}
$$

Finally, we evaluate $\mathcal{I}\left(x_{0}, u_{0}, c, u_{c}, b\right)=1$ only if any relaxation slack variable has a non-zero optimal value, as per the solution of problems $(19, \sqrt{23})$ and $(24,26)$ under the working and failing behaviors of corrective controls respectively.

\section{Chance constraint evaluation}

This module serves to evaluate the status of the chance constraint $(10)$ by aggregating the contingency analyses outcome over all contingencies $c \in \mathcal{C}$ and corrective control outcomes $b \in \mathcal{B}$. In the event that the said constraint holds true (i.e., the deterministic-constrained SCOPF solution is, as per the current partitioning of the contingency set, a feasible solution of the chance-constrained problem) the algorithm terminates. In an opposite case, the workflow advances to the contingency filtering module.

\section{E. Contingency filtering}

In order to progressively partition set $\mathcal{C}$ in a way that implies the attainability of (8), at each iteration we seek to increase the probability of maintaining the system operational limits. To do so, we search between those contingencies leading to operational constraint violations (either while not being covered by preventive and corrective controls or due to the potential failure of corrective controls) and select a contingency to be secured with greater confidence (i.e., a contingency whose contribution to the left-hand-side of (8) should be increased). To select such contingency, we explore in this work the following three contingency filtering variants.

1) Probability-based $(\mathrm{Pb})$ : this filtering variant prioritizes to address, per iteration, the most probable constraintviolating contingency. In other words, the filtered contingency is the one contributing the most to the probability summation in the left-hand-side of (8), as measured by,

$$
P b_{c}=\pi_{c} \cdot \sum_{b \in \mathcal{B}} \pi_{c}^{b} \cdot \mathcal{I}\left(x_{0}, u_{0}, c, u_{c}, b\right) .
$$

2) Feasibility-based $(\mathrm{Fb})$ this filtering variant prioritizes to address, per iteration, the most problematic constraintviolating contingency as measured by the aforementioned feasibility assessment sub-problems. That is, the filtered contingency is the one with the largest conditional infeasibility indicator, expressed as,

$$
F b_{c}=\mathbf{1}^{T}\left(\delta_{c}^{s}+\sum_{b \in \mathcal{B}} \pi_{c}^{b} \delta_{c}^{b}\right) .
$$

${ }^{4}$ Likewise, by default $\delta_{c}^{f, \star}=0, \quad \forall c \in \mathcal{C}_{\mathcal{P}}$. We also exclude from this secondary check any contingency already identified as problematic from the solution of sub-problem $19-23$, adopting by convention $\delta_{c}^{f, \star}=0$. It should also be noted that if minimization objective (13) associates a strictly positive cost to any post-contingency corrective action and problem 13 is solved to global optimality, the inspection of its solution suffices to identify contingencies in $\mathcal{C}_{\mathcal{C}}$ requiring corrective controls without checking 24,26 .
3) Risk-based $(\mathrm{Rb})$ : this filtering variant blends the former two indicators to rely on the risk implied by any contingency, quantified in terms of expected post-contingency infeasibility as,

$$
R b_{c}=\pi_{c} \cdot\left(\mathbf{1}^{T}\left(\delta_{c}^{s}+\sum_{b \in \mathcal{B}} \pi_{c}^{b} \delta_{c}^{b}\right)\right) .
$$

In the event that the selected contingency is found to be unmanageable by corrective controls, we add it to the subset $\mathcal{C}_{\mathcal{C}}$ of those contingencies to be explicitly covered by the corrective and preventive controls of the deterministicconstrained SCOPF. Alternatively, if the selected contingency is already covered (explicitly or implicitly) by corrective and preventive controls, we enhance conservativeness by imposing that it should only be covered by preventive controls and add it to the respective subset $\mathcal{C}_{\mathcal{P}}$.

\section{CAse studies}

This section presents a set of case studies on the 3-area version of the well-studied RTS-96 [23].

\section{A. Base case}

All base case data conform to the original system documentation with the exception of (i) the corrective control failure probability which is assumed equal to 0.01 , and, (ii) the generation cost function coefficients which are adopted from [24]. In similarity to the European context and without loss in generality, the cost function measures upward deviations in the generators active power output with respect to an assumed market-based dispatch $5^{5}$ To establish the latter, we solve a DCOPF problem subject to transmission capacity constraints on the cross-area interconnections only (i.e., treating each area of the system as a separate zone) and while assuming that all generating units are online.

Concerning the system operational constraints, we enforce nodal voltage limits of $[0.95,1.05]$ and the continuous ratings of all branches (column 11, in table 12 of [23]) at the preventive and corrective stages of the SCOPF problem. For the intermediate post-contingency stage, the former is relaxed to $[0.9,1.1]$ and the latter as per the short-term ratings (column 13, in table 12 of [23]). Moreover, we maintain the voltage angle difference limit across any branch at $30^{\circ}$ for all problem stages. Active and reactive power generation remains limited by the respective lower \& upper bounds. The active power ramping constraints between the preventive and corrective problem stages are computed for a 15 minute ramping interval, while we adopt the coefficients found in [25] to distribute the active power slack generation across all units at the intermediate post-contingency stage.

Finally, the contingency set $\mathcal{C}$ under consideration is composed of 111 single branch outages, discarding 9 single branch

\footnotetext{
${ }^{5}$ Notice that the choice of this objective is merely for demonstration and that the use of any other cost function, measuring for instance costs and payments to upward and downward re-dispatch actions respectively is admissible in the solution framework.
} 
TABLE I

DETERMINISTIC-CONSTRAINED SCOPF SOLUTION

\begin{tabular}{c|c}
\hline Total Cost (\$) & 881.62 \\
Explicit Contingency Set & A27;B27;C23;C27 \\
Correctively Secured Contingencies (\%) & 45 \\
\hline
\end{tabular}

outages whose practical resolution goes beyond the demonstrative scope of the present case study discussion ${ }^{6}$ By default, and unless otherwise specified, we compute the respective contingency occurrence probabilities as per the permanent forced outage rates (column 5, in table 12 of [23]) for a period of 1-hour.

To facilitate the comprehension of forthcoming results, Table [ s summarizes the solution of the deterministic-constrained SCOPF statement $13-18$ for the problem instance at hand, and while explicitly securing any contingency in corrective mode. The $1^{\text {st }}$ row lists the total (i.e. preventive and expected corrective) costs while the $2^{\text {nd }}$ presents the branches whose outage would be explicitly secured in order to identify an operational state wherein all considered contingencies are manageable, at least via corrective controls. Explicitly covering these 4 contingencies, $45 \%$ of all contingencies would be manageable by preventive and corrective controls (as shown in the $3^{\text {rd }}$ row of Table II while preventive controls alone suffice to secure $55 \%$ of all contingencies. Further, we have evaluated this solution a posteriori and found the probability of maintaining the system within all operational limits (i.e., the value constrained by (8) to be equal to $1.91 \cdot 10^{-5}$. We accordingly set the chance-constraint limit to the marginally more restrictive value of $\epsilon=10^{-5}$, implying that the direct solution of the deterministic-constrained problem is not feasible for the chance-constrained one.

a) Chance-vs deterministic-constrained reliability management: Tables $\Pi \mathrm{a}-\mathrm{c}$ present the overview of the solutions to the chance-constrained approach, as obtained by applying all three alternative contingency filtering variants. Prior to discussing differences between these three feasible solutions (and the implications of the different filtering variants), we begin by interpreting the common difference with respect to the deterministic-constrained approach in Table I

It is apparent that (i) explicitly accounting for the potential failure of corrective controls, and (ii) imposing a restrictive chance constraint means seeking an enhanced guarantee on maintaining the system operability. To achieve this guarantee, all chance-constrained solutions cover a smaller part of the contingency space with corrective controls in comparison to the deterministic-constrained solution. Indeed, the $3^{\text {rd }}$ rows of Tables П $\mathrm{a}-\mathrm{c}$ exhibit that arriving at a feasible solution with respect to the chance constraint necessitates explicitly covering a certain sub-set of contingencies in preventive mode only. Further, this shift of the operating state has an effect over

\footnotetext{
${ }^{6}$ Such as outages leading to islanding, insufficient nodal reactive power compensation capacity etc.. Notice also that while our case study focuses on the reliability of the transmission sub-system, the proposed solution approach and our developed implementation involves no inherent restriction to the consideration of contingencies on the generation sub-system.
}

TABLE II

CHANCE-CONSTRAINED SCOPF SOLUTION

\begin{tabular}{c|c}
\hline \multicolumn{2}{c}{ a. Probability-based } \\
\hline Total Cost (\$) & 882.37 \\
Chance-constraint level & $9.85 \mathrm{e}-6$ \\
\hline Explicit Corrective Contingency Set & A25-2;;A27;B26;B27 \\
C23;C25-2;C27 \\
Explicit Preventive Contingency Set & A30;B21;B22;B30;C21;C30 \\
Correctively Secured Contingencies (\%) & 26.12 \\
\hline \multicolumn{2}{c}{ b. Feasibility-based } \\
\hline Total Cost (\$) & 896.78 \\
Chance-constraint level & $5.28 \mathrm{e}-6$ \\
\hline Explicit Corrective Contingency Set & A27;B27;C23;C27 \\
Explicit Preventive Contingency Set & B13-2 \\
Correctively Secured Contingencies (\%) & 11.71 \\
\hline \multicolumn{2}{c}{ c. Risk-based } \\
Total Cost (\$) \\
Chance-constraint level \\
\hline Explicit Corrective Contingency Set \\
Explicit Preventive Contingency Set \\
Correctively Secured Contingencies (\%) \\
\hline
\end{tabular}

the whole contingency space. This effect is showcased in the $4^{\text {th }}$ rows of Tables $\amalg \mathrm{a}-\mathrm{c}$ wherein for all feasible solutions the balance of interest is adjusted in favor of preventively secured contingencies with respect to the last row in Table [1. Using corrective controls against fewer contingencies, it is more probable that the post-contingency constraints will be valid since preventive controls are fully fail-safe.

The trade-off between preventive and corrective controls is not only a matter of security but also a matter of efficiency. The more secure chance-constrained solutions are as anticipated slightly more expensive than the deterministic-constrained one (as seen in the $1^{\text {st }}$ rows of Tables $\Pi \mathrm{a}-\mathrm{c}$ ), recalling that we have intentionally selected a more restrictive limit for the sake of the intended demonstration 7 To give the complete perspective, it is worthwhile to mention here that we have also solved the preventive only variant of the deterministicconstrained problem (i.e., fully excluding corrective controls and only using subset $\mathcal{C}_{\mathcal{P}}$ to define contingencies to be explicitly secured) at cost of $962.03 \$$. In other words, the chanceconstrained SCOPF enables accessing intermediate solutions between the cheaper, less fail-safe preventive \& corrective deterministic-constrained approach and its more expensive, more conservative counterpart.

b) The alternative contingency filtering variants: Moving further, let us focus on the three algorithmic variants distinguished by the metric used for contingency filtering. Firstly, we notice from Table $\Pi$ that the probability- and riskbased filtering variants essentially return a common solution in terms of total cost, chance constraint level and percentage of correctively secured contingencies. In addition, the sets of explicitly covered contingencies identified by the risk-based

\footnotetext{
${ }^{7}$ Setting such limit in practice is of course a topic which merits a detailed socio-economic assessment beyond the scope of the present case studies [26].
} 
TABLE III

COMPUTATIONAL TIME BREAKDOWN (SEC)

\begin{tabular}{c|c|c|c}
\hline Step $\backslash$ Filter & Probability & Feasibility & Risk \\
\hline SCOPF & 121.48 & 16.48 & 23.07 \\
Contingency Analysis & 2123.18 & 694.51 & 818.77 \\
Contingency Filtering & 0.002 & 0.0001 & 0.0008 \\
Chance Constraint & 0.06 & 0.003 & 0.003 \\
\hline Total & 2244.67 & 711.02 & 841.84 \\
\hline
\end{tabular}

variant $\left(3^{\text {rd }}\right.$ and $4^{\text {th }}$ row in Table II c) are respective subsets of those identified by the probability-based variant $\left(3^{\text {rd }}\right.$ and $4^{\text {th }}$ row in Table II a). Putting all these evidence together, we argue that the risk-based variant is algorithmically more efficient with respect to the probability-based one. The issue with the latter appears to be that, while the magnitude of the contingency probabilities is indeed critical to achieving constraint (8), neglecting the severity dimension may lead to initially filtering contingencies that are not in the active set of the final feasible solution. This is of course attributable to the fact that the contingency and corrective control failure occurrence probabilities are predefined parameters of the optimization problem at hand.

The prominence of the severity dimension for algorithmic efficiency is further supported by the results pertaining to the feasibility-based filtering variant, Table $\Pi$ b. Indeed, this filtering variant results in identifying explicit preventive and corrective contingency sets of the smallest cardinality. The distinctive characteristic here is, as anticipated, conservativeness reflected both in the chance constraint level as well as in the smaller percentage of correctively secured contingencies. Inevitably, the price to pay for the conservativeness is also reflected in the total cost value which is slightly increased with respect to the feasible solution provided by the riskand probability-based variants. Noting the strong similarity in the contingencies filtered by the feasibility- and risk- based variants, we have also studied the detailed filtering sequences (that is, order of contingencies added to subsets $\mathcal{C}_{\mathcal{C}}, \mathcal{C}_{\mathcal{P}}$ from the start to the end of the algorithm). Our records show that both approaches identify the same four contingencies as the deterministic-constrained approach (i.e., those shown in the $2^{\text {nd }}$ row of Table If at their four first iterations. At the subsequent fifth iteration, the choice of the feasibility-based scheme is more effective in terms of enhancing the guarantee of operability, but also more costly to secure.

c) Computational performance properties: Our implementation of the proposed heuristic algorithm was developed in Julia [27] while using the modelling language JuMP [28] as well as the PowerModels.jl framework [29]. Further, we employed IPOPT [30] as the solver for all OPF/SCOPF problem instances. The hardware used features a 4-core, 4.2 $\mathrm{GHz}$ processor and $24 \mathrm{~GB}$ of RAM.

Table III reports the breakdown of the computational time to solve the 'base-case' instance using all three contingency filtering variants under study. As anticipated, the major workload is the 'contingency analysis' step of the algorithm, which consists of solving, per contingency, the feasibility checking AC-OPF problems (19, 23) and, if needed, 24 26). More specifically, $64.45 \%$ of the total time for contingency analysis reported in the $2^{\text {nd }}$ row of Table III was spent solving instances of problem (19, 23) to evaluate the feasibility of post-contingency stages under the assumption that corrective controls would work. Respectively, $35.55 \%$ of the time was spent solving instances of problem (24-26) to quantify the impact of potential failures of corrective controls. Moreover, $51.6 \%$ of the total number of AC-OPF problems solved for contingency analysis were instances of (19) while $48.4 \%$ were instances of 24 26). Finally, over the different filtering approaches and algorithmic iterations, the average time needed to solve (19, 23) and 24 26) for a single contingency was 0.86 seconds and 0.39 seconds respectively.

We must underline here that, for the demonstrative purpose of our implementation, we performed the operations solving feasibility assessment problems $19 \sqrt{23}$ and 24,26 in sequence over the set of contingencies under consideration. However, there are no input/output dependencies between the problem instances referring to different contingencies. Exploiting parallelization to achieve enhanced computational performance while solving the, separable over different contingencies, problems (19, 23) and 24 26) is an obvious strategy for a practical implementation of the heuristic algorithm, in a similar manner to [15]. We refer the reader to [5] for an indication of the potential gains in computational performance of a parallel implementation of this step.

d) Sensitivity analysis: To detail the difference between the alternative contingency filtering options, Tables IV and $\mathrm{V}$ present sensitivity analyses with respect to the threshold of the chance constraint as well as the corrective control failure probability. Firstly, it is apparent from both sets of results that the feasibility- and risk-based filtering methods consistently outperform the probability-based one. As the chance threshold becomes more restrictive, Table IV] shows that the cost advantage of the risk- over the feasibility-based solution diminishes. Moreover, the feasibility-based filtering method identifies a feasible solution in fewer iterations, which in turn results in reduced computational time. To interpret such results, we underline that a more stringent chance constraint threshold means seeking for a more conservative SCOPF solution of greater reliance on the fail-safe preventive controls. In such conditions, the more conservative feasibility-based filtering appears more efficient. Similar effects can also be identified through the results of Table $\mathrm{V}$. For a fixed chance constraint threshold, an increase in the probability of failure of corrective controls implies again a need for a more conservative SCOPF solution, since the margin for using the riskier corrective controls is smaller. As previously, the feasibility-based filtering performs marginally more efficiently than the risk-based one, while the probability-based filtering takes considerably more iterations to identify costlier feasible solutions.

\section{B. Impact of weather-dependent contingency probabilities}

Noting from the base case that the differences between the feasibility- and risk-based approaches appear marginal, 
TABLE IV

SENSitivity ANALYSiS - CHANCE THRESHOLD $(\epsilon)$

\begin{tabular}{c|c|c|c}
\hline$(\epsilon) \backslash$ Filter & Probability & Feasibility & Risk \\
\hline \multicolumn{4}{c}{ (a) Total cost (\$) } \\
\hline 5 E-5 & 881.62 & 881.62 & 881.62 \\
E-5 & 882.37 & 896.78 & 882.37 \\
$7.5 E-5$ & 896.78 & 896.78 & 896.78 \\
$2.5 E-6$ & 957.33 & 902.68 & 905.00 \\
E-6 & 957.97 & 944.93 & 944.93
\end{tabular}

(b) Iterations

\begin{tabular}{c|c|c|c}
\hline $5 \mathrm{E}-5$ & 10 & 5 & 5 \\
$\mathrm{E}-5$ & 16 & 6 & 7 \\
$7.5 \mathrm{E}-5$ & 17 & 6 & 8 \\
$2.5 \mathrm{E}-6$ & 23 & 11 & 14 \\
$\mathrm{E}-6$ & 24 & 16 & 17 \\
\hline
\end{tabular}

(c) Computational time (sec)

\begin{tabular}{c|c|c|c}
$5 \mathrm{E}-5$ & 1372.25 & 738.84 & 610.94 \\
E-5 & 2244.67 & 711.02 & 841.84 \\
$7.5 \mathrm{E}-5$ & 2317.58 & 710.28 & 962.84 \\
$2.5 \mathrm{E}-6$ & 2999.03 & 1272.78 & 1639.21 \\
E-6 & 3073.27 & 1816.46 & 1960.53
\end{tabular}

TABLE V

SENSITIVITY ANALYSIS - CORRECTIVE FAILURE PROBABILITY $\left(\pi_{c}^{f}\right)$

\begin{tabular}{c|c|c|c}
\hline$\left(\pi_{c}^{f}\right) \backslash$ Filter & Probability & Feasibility & Risk \\
\hline \multicolumn{4}{|c}{ (a) Total cost (\$) } \\
\hline E-3 & 881.62 & 881.62 & 881.62 \\
$5 E-3$ & 881.62 & 881.62 & 881.62 \\
E-2 & 882.37 & 896.78 & 882.37 \\
$2.5 E-2$ & 901.97 & 899.62 & 899.62 \\
$5 E-2$ & 957.33 & 902.68 & 905.00 \\
\hline
\end{tabular}

(b) Iterations

\begin{tabular}{c|c|c|c}
\hline E-3 & 10 & 5 & 5 \\
$5 \mathrm{E}-3$ & 11 & 5 & 5 \\
$\mathrm{E}-2$ & 16 & 6 & 7 \\
$2.5 \mathrm{E}-2$ & 20 & 7 & 9 \\
$5 \mathrm{E}-2$ & 23 & 10 & 13 \\
\hline \multicolumn{3}{c}{ (c) Computational time (sec) } \\
\hline E-3 & 1212.13 & 602.21 & 602.60 \\
$5 \mathrm{E}-3$ & 1341.31 & 610.31 & 611.08 \\
$\mathrm{E}-2$ & 2244.67 & 711.02 & 841.84 \\
$2.5 \mathrm{E}-2$ & 2403.31 & 833.80 & 1070.46 \\
$5 \mathrm{E}-2$ & 2750.48 & 1278.43 & 1502.15 \\
\hline
\end{tabular}

while the probability-based filtering method is markedly less efficient, we study in greater detail the difference between the former two on an extended set of cases reflecting the variability of contingency probabilities in function of the weather conditions. To facilitate this analysis, we model the effect of the weather state on outage rates (hence, outage probabilities) on the basis of [31]. More specifically, we adopt a two state (i.e., normal vs adverse) weather model while assuming that: i) $60 \%$ of recorded failures occur under adverse weather and, ii) the weather state is normal for $90 \%$ of a typical year. We define the three additional case studies by assuming that only
TABLE VI

FEASibility-BASED FILTERING VS AdVERSE WEATHER CASES

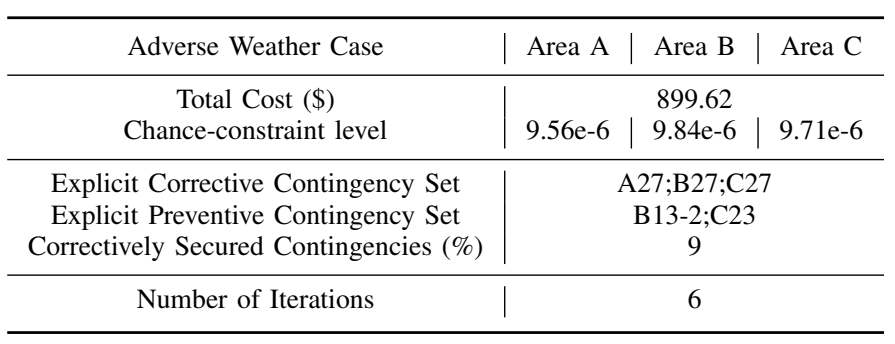

TABLE VII

Risk-BASEd FiLtering CONTINGENCY MANAGEMENT

\begin{tabular}{cc|c|c|c}
\hline \multicolumn{2}{c|}{ Adverse Weather Case } & Area A & Area B & Area C \\
\hline \multirow{2}{*}{ In Area A } & Correctively Secured (\%) & 0 & 9 & 9 \\
& Preventively Secured (\%) & 100 & 91 & 91 \\
\hline \multirow{2}{*}{ In Area B } & Correctively Secured (\%) & 0 & 0 & 9 \\
& Preventively Secured (\%) & 100 & 100 & 91 \\
\hline \multirow{2}{*}{ In Area C } & Correctively Secured (\%) & 9 & 14 & 12 \\
& Preventively Secured (\%) & 91 & 86 & 88 \\
\hline
\end{tabular}

one of the three system areas faces adverse weather (implying increased probability of realizing a contingency) while the weather state is normal for the remaining two areas.

The feasibility-based filtering approach is in essence unaffected by the studied variations in contingency probabilities in terms of the filtering sequence it yields (i.e., the order of choosing contingencies to be explicitly covered by corrective and/or preventive controls). In these simulations, it also converges on the same feasible solution irrespective of which area is exposed to the adverse weather conditions. The common solution overview is provided in Table VI wherein the slight variation in the probability of maintaining the system functionality shown in the $2^{\text {nd }}$ row is attributable to the variations in the contingency probabilities 8 The choices between preventive and corrective controls are also quite balanced across all three areas of the system. More specifically, 9\% of contingencies in each area being secured by corrective controls (either implicitly or explicitly), while all cross-area interconnections are secured preventively. Recalling the strong similarities in topology, generation and load across the 3 areas of the test system, such balance is in fact anticipated. Finally, we note that with respect to the base-case set-up of subsection IV-A and the solution presented in Table $\Pi \mathrm{b}$, the relative increase in the probability of realizing a contingency results in greater cautiousness manifested in explicitly securing the outage C23 in preventive rather than corrective mode, and in securing a smaller percentage of contingencies by corrective controls.

Table VII presents the partitioning of preventively and correctively secured contingencies per area as per the solutions

\footnotetext{
${ }^{8} \mathrm{~A}$ similar slight variation in the total cost, due to the impact of contingency probability on the corrective control cost expectation is below the adopted rounding accuracy hence not observable in the cost values listed here.
} 
TABLE VIII

RISK-BASED FILTERING CONTINGENCY SEQUENCE

\begin{tabular}{c|c|c|c}
\hline \multicolumn{1}{|c|}{ Adverse Weather Case } & Area A & Area B & Area C \\
\hline Step 1 & A27 (Cor.) & B23 (Cor.) & C27 (Cor.) \\
Step 2 & A23 (Cor.) & B27 (Cor.) & C23 (Cor.) \\
Step 3 & A30 (Cor.) & B21 (Cor.) & C30 (Cor.) \\
Step 4 & A21 (Prev.) & B22 (Cor.) & C21 (Prev.) \\
Step 5 & A26 (Prev.) & B13-2 (Prev.) & C26 (Prev.) \\
Step 6 & A23 (Prev.) & A27 (Cor.) & C23 (Prev.) \\
Step 7 & C27 (Cor.) & C27 (Cor.) & A27(Cor.) \\
Step 8 & A13-2 (Prev.) & C23(Cor.) & B27 (Cor.) \\
Step 9 & A24 (Cor.) & B27 (Prev.) & B13-2 (Prev.) \\
Step 10 & B13-2 (Cor.) & & \\
Step 11 & C23 (Cor.) & & \\
\hline
\end{tabular}

TABLE IX

Risk-BASEd Filtering Vs Adverse Weather Cases

\begin{tabular}{c|c|c|c}
\hline Adverse Weather Case & Area A & Area B & Area C \\
\hline Total Cost (\$) & 941.04 & 897.09 & 899.62 \\
Chance constraint level & $5.67 \mathrm{e}-7$ & $1.32 \mathrm{e}-6$ & $9.71 \mathrm{e}-6$ \\
\hline Number of Iterations & 11 & 9 & 9 \\
\hline
\end{tabular}

obtained while applying the risk-based filter. Let us first notice how in both cases wherein the adverse weather affects areas A or B (shown in the $1^{\text {st }}$ and $2^{\text {nd }}$ columns of Table VII) all contingencies inside the area facing adverse weather would be secured by preventive controls. The interpretation is that the increased likelihood of realizing a contingency stimulates conservativeness. Further, Table VIII] lists the filtering sequences of explicitly covered contingencies. Each column of this table presents the filtering sequence corresponding to the case wherein the respective area is facing adverse weather conditions, while the abbreviations within the parenthesis next to each contingency ID denote whether it is to be explicitly covered by corrective (Cor.) or only preventive (Prev.) controls. We recall here that the latter choice depends on the status of the filtered contingency and that a filtered contingency may only be designated to be explicitly covered by preventive controls if it is already covered (explicitly or implicitly) by corrective controls. We observe that in all cases studied, contingencies within the respective adverse weather area are (i) the ones to be filtered first, and (ii) the ones composing the largest part of the respective subsets.

Finally, Table IX lists the cost values as well as the number of iterations taken by the risk-based heuristic algorithm to solve the cases studied. Concerning economic cost, the risk-based approach was found to marginally outperform the feasibility-based filtering in one test case, while being more notably inferior in another test-case. The difference is more striking in terms of the algorithmic efficiency of the heuristic approach, wherein feasibility-based filtering shows a more concrete advantage in terms of fewer iterations.

\section{CONCLUSIONS \& FUTURE WORK}

This paper revisited the fundamental SCOPF problem of safeguarding the operability of the power system in light of the potential development of contingency events. The modeling scope goes beyond the current standard by taking into account uncertainty on the effectiveness of post-contingency corrective controls, whose potential malfunctioning may escalate a single outage into a catastrophic cascading sequence of failures. The extended SCOPF statement achieves an explicit guarantee on the probability of maintaining the system within all operational limits. Combining such extension with the AC power flow model in its full form yields a non-convex Mixed Integer Nonlinear Programming (MINLP) problem, featuring a coupling constraint on all recourse decision stages as well as disjunctive inequalities. To tackle it, we adopted the realistic prioritization of feasibility before optimality [32] and developed a workable heuristic approach progressively shrinking the feasible region of a (conventional) deterministic-constrained SCOPF problem.

Keeping in mind the hurdles in the ongoing transition towards an explicitly probabilistic approach to reliability management, we placed emphasis on establishing interpretability as the main feature of our proposal. We have showcased here the essential functionality of the chance constraint in enforcing a certain level of confidence on the trade-off between preventive and corrective controls. It may imply reducing the number of contingencies secured by means of corrective controls to guarantee that the probability of yielding operational limit violations (potentially in turn triggering cascading events) is indeed low enough. Alternatively, it may be understood as relaxing the conservativeness of the preventive only approach, which, given the corrective control effectiveness uncertainty, is truly the only strategy offering full certainty but at the expense of significantly increased economic costs.

\section{A. Towards enhanced scalability \& algorithmic efficiency}

We intentionally opted for a practical solution approach, targeting feasibility and achievable with marginal modifications on existing tools and overarching solution strategies. Our goal was to show that existing practices can be leveraged to find feasible solutions for the chance-constrained statement, thus unlocking a first step for the adoption of probabilistic methods. We must however recognize the ample scope for improvements to address the size and computational performance requirements of large-scale power systems. Regarding contingency analysis, i.e. the major workload in our implementation, solving the feasibility assessment problems $(19,23)$ and, if needed, 2426 in series over the set of postulated contingencies is only sufficient for a demonstrative scope. Notice however that there are no input/output dependencies between the feasibility assessment problems referring to different contingencies. Thus, for a practical implementation, it is straightforward to solve the feasibility assessment problems 19,23 and 24 26) in parallel for different contingencies. In our future work, we shall also explore the use of machine learning techniques to build predictors for the optimal objective value of these problems, thus significantly reducing the computational time requirement of this step. We point to [33], [34] for early findings on the potential to build and exploit such predictors and also to [35], [36] further establishing the viability of relying on machine learning to enable faster contingency 
screening. Let us not undermine the challenge of solving the deterministic-constrained problem (13,18) vs a large-scale power system though. While the problem size we tackled here remains directly manageable by a standard solver, we already foresee integrating state-of-the-art enhancements from the literature for improved scalability. In particular, we regard the approach of combining contingency filtering with network reduction introduced in [15] as the established feasible strategy for solving the contingency constrained problem at the scale of the European network.

\section{B. Towards the practical adoption of probabilistic methods for reliability management}

Further from the implementation of the basic framework in an more advanced computational setup, the systematic collection of additional data as well as the integration of SCOPF tools (such as our proposal) in operational \& planning practices are foreseeable future steps towards realizing a transition to probabilistic reliability management. Concerning the former issue the notable gap relates to the data needed to accurately quantify the probability of failure for postcontingency corrective controls. We refer the reader to [9], [37] for the alternatives that could be used to fill the data gap, including periodic inspections of the functionality of the involved controllable devices as well as capitalizing on the intuition and experience of control room operators. Regarding the integration of SCOPF into operational \& planning practices, we observe with great interest recent initiatives such as the so-called "grid optimization competition" [25]. Reference [38] presents a comprehensive discussion on the relevance of advanced optimization concepts for the operation \& planning of the modern grid, while the transition roadmap presented in [1] also foresees the potential role of SCOPF tools in the future of practical reliability management.

\section{Towards a unified framework in planning \& operation}

Last but not least, we underline the need to progressively develop a unified framework for power system reliability management through planning and operation [17]. In this direction, the next step of our work is the revision of the socalled "look-ahead" reliability management problem, featuring a more extended decision horizon including a sequence of instances of the fundamental problem addressed here, as well as uncertainty on nodal power injections and loads (for instance due to renewable power generation) and potentially on the weather state affecting contingency probabilities. At the conceptual level, the extension of this framework to also address such planning problems and the additional uncertainties is developed in [39].

\section{REFERENCES}

[1] GARPUR Consortium, "A transition roadmap towards probabilistic reliability management," 7th framework programme, EU Commission grant agreement 608540, Oct. 2017. [Online]. Available: https: //www.sintef.no/projectweb/garpur/deliverables/
[2] F. Capitanescu, "Critical review of recent advances and further developments needed in AC optimal power flow," Electric Power Systems Research, vol. 136, pp. $57-68,2016$.

[3] Y. Xu, Z. Y. Dong, R. Zhang, K. P. Wong, and M. Lai, "Solving preventive-corrective SCOPF by a hybrid computational strategy," IEEE Transactions on Power Systems, vol. 29, no. 3, pp. 1345-1355, May 2014.

[4] F. Capitanescu, "Enhanced risk-based SCOPF formulation balancing operation cost and expected voluntary load shedding," Electric Power Systems Research, vol. 128, pp. 151 - 155, 2015.

[5] D. Shchetinin and G. Hug, "Decomposed algorithm for risk-constrained AC OPF with corrective control by series FACTS devices," Electric Power Systems Research, vol. 141, pp. 344 - 353, 2016.

[6] Q. Wang, J. D. McCalley, T. Zheng, and E. Litvinov, "Solving corrective risk-based security-constrained optimal power flow with Lagrangian relaxation and Benders decomposition," International Journal of Electrical Power \& Energy Systems, vol. 75, pp. 255 - 264, 2016.

[7] GARPUR Consortium, "Current practices, drivers and barriers for new reliability standards," 7th framework programme, EU Commission grant agreement 608540, 2014. [Online]. Available: https://www.sintef.no/projectweb/garpur/deliverables/

[8] K. Yamashita, J. Li, P. Zhang, and C. Liu, "Analysis and control of major blackout events," in 2009 IEEE/PES Power Systems Conference and Exposition, March 2009, pp. 1-4.

[9] V. V. Vadlamudi, C. Hamon, O. Gerde, G. Kjølle, and S. Perkin, "On improving data and models on corrective control failures for use in probabilistic reliability management," in Probabilistic Methods Applied to Power Systems (PMAPS), 2016 International Conference on. IEEE, 2016, pp. 1-6.

[10] E. Karangelos and L. Wehenkel, "Post-contingency corrective control failure - a risk to neglect or a risk to control?" in Probabilistic Methods Applied to Power Systems (PMAPS), 2018 International Conference on. IEEE, 2018, pp. 1-6.

[11] E. Karangelos, P. Panciatici, and L. Wehenkel, "Whither probabilistic security management for real-time operation of power systems ?" in Bulk Power System Dynamics and Control (iREP) - IX (iREP), 2013 iREP Symposium, 2013.

[12] E. Karangelos and L. Wehenkel, "Probabilistic reliability management approach and criteria for power system real-time operation," in 2016 Power Systems Computation Conference (PSCC), June 2016, pp. 1-9.

[13] F. Capitanescu and L. Wehenkel, "A new iterative approach to the corrective security-constrained optimal power flow problem," IEEE Transactions on Power Systems, vol. 23, no. 4, pp. 1533-1541, Nov 2008.

[14] A. Marano-Marcolini, F. Capitanescu, J. L. Martinez-Ramos, and L. Wehenkel, "Exploiting the use of DC SCOPF approximation to improve iterative AC SCOPF algorithms," IEEE Transactions on Power Systems, vol. 27, no. 3, pp. 1459-1466, Aug 2012.

[15] L. Platbrood, F. Capitanescu, C. Merckx, H. Crisciu, and L. Wehenkel, "A generic approach for solving nonlinear-discrete security-constrained optimal power flow problems in large-scale systems," IEEE Transactions on Power Systems, vol. 29, no. 3, pp. 1194-1203, May 2014.

[16] F. Capitanescu, J. Martinez Ramos, P. Panciatici, D. Kirschen, A. Marano Marcolini, L. Platbrood, and L. Wehenkel, "State-of-theart, challenges, and future trends in security constrained optimal power flow," Electric Power Systems Research, vol. 81, no. 8, pp. 1731-1741, 2011.

[17] GARPUR Consortium, "Guidelines for implementing the new reliability assessment and optimization methodology," 7th framework programme, EU Commission grant agreement 608540, Nov. 2016. [Online]. Available: https://www.sintef.no/projectweb/garpur/deliverables/

[18] R. Billinton and R. N. Allan, Reliability Evaluation of Power Systems, 2nd ed. New York: Plenum, 1996.

[19] F. Capitanescu and L. Wehenkel, "Redispatching active and reactive powers using a limited number of control actions," IEEE Transactions on Power Systems, vol. 26, no. 3, pp. 1221-1230, Aug 2011.

[20] B. Zeng and L. Zhao, "Solving two-stage robust optimization problems using a column-and-constraint generation method," Operations Research Letters, vol. 41, no. 5, pp. 457 - 461, 2013.

[21] A. Komen, "Benders decomposition vs column \& constraint generation, a closer look," Utrecht University, 2017.

[22] M. Ruiz, J. Maeght, A. Marié, P. Panciatici, and A. Renaud, "A progressive method to solve large-scale AC optimal power flow with discrete variables and control of the feasibility," in 2014 Power Systems Computation Conference, Aug 2014, pp. 1-7.

[23] C. Grigg, P. Wong, P. Albrecht, R. Allan, M. Bhavaraju, R. Billinton, Q. Chen, C. Fong, S. Haddad, S. Kuruganty, W. Li, R. Mukerji, 
D. Patton, N. Rau, D. Reppen, A. Schneider, M. Shahidehpour, and C. Singh, "The IEEE Reliability Test System-1996. A report prepared by the Reliability Test System Task Force of the Application of Probability Methods Subcommittee," IEEE Transactions on Power Systems, vol. 14 , no. 3, pp. 1010-1020, Aug 1999.

[24] C. Coffrin, "A Library of IEEE PES Power Grid Benchmarks," https: //power-grid-lib.github.io/. [Online; accessed 19-July-2018].

[25] "Grid Optimization Competition," https://gocompetition.energy.gov/ content/grid-optimization-competition [Online; accessed 19-July-2018].

[26] GARPUR Consortium, "Recommendations for implementing the socio-economic impact assessment methodology over the paneuropean system in a tractable way," 7th framework programme, EU Commission grant agreement 608540, 2016. [Online]. Available: https://www.sintef.no/projectweb/garpur/deliverables/

[27] J. Bezanson, A. Edelman, S. Karpinski, and V. Shah, "Julia: A fresh approach to numerical computing," SIAM Review, vol. 59, no. 1, pp. 65-98, 2017. [Online]. Available: https://doi.org/10.1137/141000671

[28] I. Dunning, J. Huchette, and M. Lubin, "JuMP: A modeling language for mathematical optimization," SIAM Review, vol. 59, no. 2, pp. 295-320, 2017. [Online]. Available: https://doi.org/10.1137/15M1020575

[29] C. Coffrin, R. Bent, K. Sundar, Y. Ng, and M. Lubin, "PowerModels.jl: An open-source framework for exploring power formulations," in 2018 Power Systems Computation Conference (PSCC), June 2018.

[30] A. Wächter and L. T. Biegler, "On the implementation of an interior-point filter line-search algorithm for large-scale nonlinear programming," Math. Program., vol. 106, no. 1, pp. 25-57, May 2006. [Online]. Available: http://dx.doi.org/10.1007/s10107-004-0559-y

[31] R. Billinton and G. Singh, "Application of adverse and extreme adverse weather: modelling in transmission and distribution system reliability evaluation," Generation, Transmission and Distribution, IEE Proceedings-, vol. 153, no. 1, pp. 115-120, Jan 2006.

[32] B. Stott and O. Alsac, "Optimal power flow - basic requirements for real-life problems and their solutions," Jan. 2012.

[33] L. Duchesne, E. Karangelos, and L. Wehenkel, "Machine learning of real-time power systems reliability management response," in 2017 IEEE Manchester PowerTech, June 2017, pp. 1-6.

[34] — " "Using machine learning to enable probabilistic reliability assessment in operation planning," in 2018 Power Systems Computation Conference (PSCC), June 2018, pp. 1-8.

[35] B. Donnot, I. Guyon, M. Schoenauer, A. Marot, and P. Panciatici, "Anticipating contingengies in power grids using fast neural net screening," in IEEE World Congress on Computational Intelligence (WCCI), July 2018.

[36] - " "Optimization of computational budget for power system risk assessment," in 2018 IEEE PES Innovative Smart Grid Technologies (ISGT) Conference Europe, July 2018.

[37] GARPUR Consortium, "Results and reccomendations towards stakeholders," 7th framework programme, EU Commission grant agreement 608540, 2017. [Online]. Available: https: //www.sintef.no/projectweb/garpur/deliverables/

[38] P. Panciatici, M. C. Campi, S. Garatti, S. H. Low, D. K. Molzahn, A. X. Sun, and L. Wehenkel, "Advanced optimization methods for power systems," in 2014 Power Systems Computation Conference, Aug 2014, pp. 1-18.

[39] E. Karangelos and L. Wehenkel, "Probabilistic reliability management approach and criteria for power system short-term operational planning," in Bulk Power System Dynamics and Control (iREP) - X (iREP), 2017 iREP Symposium, 2017.

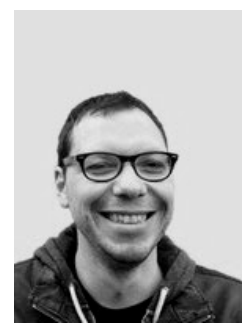

Efthymios Karangelos (M'13) received the Diploma degree in mechanical engineering from the National Technical University of Athens, Athens, Greece, in 2005, and the M.Sc. degree in power systems engineering and economics and the $\mathrm{Ph} . \mathrm{D}$. degree in electrical engineering from the University of Manchester, Manchester, U.K., in 2007 and 2012, respectively. In 2012, he joined the Department of Electrical Engineering and Computer Science, University of Liège, Liège, Belgium, as a Post-Doctoral Researcher. His current research interests include power system modeling, reliability and risk management and stochastic optimization.

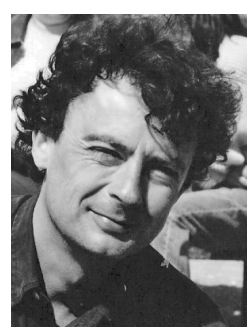

Louis Wehenkel graduated in Electrical Engineering (Electronics) in 1986 and received the Ph.D. degree in 1990, both from the University of Liège, where he is full Professor of Electrical Engineering and Computer Science. His research interests lie in the fields of stochastic methods for systems control, optimization, machine learning and data mining, with applications in complex systems, in particular large scale power systems planning, operation and control, industrial process control, bioinformatics and computer vision. 